\title{
FINITENESS OF INDEX AND TOTAL SCALAR CURVATURE FOR MINIMAL HYPERSURFACES
}

\author{
JOHAN TYSK
}

(Communicated by David G. Ebin)

\begin{abstract}
Let $M^{n}, n \geq 3$, be an oriented minimally immersed complete hypersurface in Euclidean space. We show that for $n=3,4,5$, or 6 , the index of $M^{n}$ is finite if and only if the total scalar curvature of $M^{n}$ is finite, provided that the volume growth of $M^{n}$ is bounded by a constant times $r^{n}$, where $r$ is the Euclidean distance function. We also note that this result does not hold for $n \geq 8$. Moreover, we show that the index of $M^{n}$ is bounded by a constant multiple of the total scalar curvature for all $n \geq 3$, without any assumptions on the volume growth of $M^{n}$.
\end{abstract}

\section{INTRODUCTION}

The index of a minimally immersed hypersurface $M^{n}$ in $R^{n+1}$ is defined to be the limit of the indices of an increasing sequence of exhausting compact domains in $M$. The index of a domain $D$ is the number of negative eigenvalues of the eigenvalue problem

$$
\left(\Delta+|A|^{2}\right) \phi+\lambda \phi=0 \text { on } D,\left.\quad \phi\right|_{\partial D}=0 .
$$

Here, $|A|$ denotes the length of the second fundamental form of $M^{n}$ as a submanifold of $R^{n+1}$. Geometrically, the index of $M$ can be described as the maximum dimension of a linear space of compactly supported deformations that decrease the volume up to second order. D. Fischer-Colbrie [2], obtains the very interesting result that a complete oriented minimal surface in $R^{3}$ has finite index if and only if it has finite total curvature. The proof of this result uses the conformal invariance of the Dirichlet integral in dimension two and does not seem to generalize to higher dimensions.

In the present paper we extend the results mentioned above to minimal hypersurfaces in $R^{4}, R^{5}, R^{6}$ and $R^{7}$, satisfying a certain volume growth condition. We also observe that the theorem does not hold in $R^{n+1}$ for $n \geq 8$. More precisely, we show the following: Let $M^{n} \subseteq R^{n+1}, n=3,4,5$, or 6 , be

Received by the editors November 26, 1986 and, in revised form, April 22, 1988.

1980 Mathematics Subject Classification (1985 Revision). Primary 53C42; Secondary 58C40.

Key words and phrases. Minimal hypersurface, index, eigenvalue, second fundamental form. 
a complete oriented minimally immersed hypersurface with

$$
\lim _{r \rightarrow \infty} \frac{\operatorname{vol}(M \cap B(r))}{r^{n}}<\infty,
$$

where $r$ is Euclidean distance and $B(r)$ is a Euclidean ball of radius $r$. Under these assumptions, we prove that $M^{n}$ has finite index if and only if $M^{n}$ has finite total scalar curvature. The total scalar curvature of $M^{n}$ is defined to be $\int_{M^{n}}|A|^{n}$, which is a generalization of the total curvature for surfaces. We call this integral total scalar curvature since for minimal submanifolds of Euclidean space, $|A|^{2}$ is equal to minus one times the scalar curvature. The volume growth condition $(*)$ is quite natural since if $\int_{M^{n}}|A|^{n}<\infty$, then (*) holds, see M. Anderson [1]. In fact, we show that the index is bounded by a multiple, depending only on the dimension $n$, of the total scalar curvature, in all dimensions $n \geq 3$ (a similar estimate also holds for $n=2$, see J. Tysk [8]). However, the converse is not true for $n \geq 8$, as mentioned above, and it is not known if finite index implies that $(*)$ is satisfied.

We would like to thank the referee for pointing out that the original formulation of the main theorem contained a redundant assumption on $M$.

\section{NOTATION, STATEMENT OF THE THEOREM, AND SOME REMARKS}

The notation to be used throughout the rest of the paper is the following:

$A=$ the second fundamental form of $M^{n}$ as a submanifold of $R^{n+1}$;

$r=$ the distance function in the ambient Euclidean space;

$B(r)=$ a ball of radius $r$ in the ambient Euclidean space;

$B_{p}(r)=$ same as above but with specified center $p$;

$D_{0}(\rho)=$ a geodesic ball in $M^{n}$ with radius $\rho$ centered at 0 ;

$\omega_{n}=$ the volume of the Euclidean unit ball in $R^{n}$.

Our main result is the following theorem.

Theorem. Let $M^{n}, n=3,4,5$, or 6 , be an oriented minimally immersed complete hypersurface in Euclidean space, satisfying the following volume growth condition

$$
\lim _{r \rightarrow \infty} \frac{\operatorname{vol}(M \cap B(r))}{r^{n}}<\infty .
$$

Then $M$ has finite index if and only if $\int_{M}|A|^{n}<\infty$.

Remarks. In $R^{9}$ and in higher dimensional Euclidean spaces there are areaminimizing graphs with infinite total scalar curvature. These minimizing graphs satisfy the volume growth condition $(*)$ and are, of course, stable, or equivalently, they have index zero, making it clear that our theorem does not generalize to these dimensions. In $R^{8}$, there are stable cones which cause our method of proof to fail. However, it is not known if there are stable nontrivial (regular) minimal hypersurfaces in $R^{8}$, or for that matter, in $R^{4}, R^{5}, R^{6}$, or $R^{7}$. Our result might therefore still be true in $R^{8}$. Another interesting question is 
whether the volume growth condition of our theorem is necessary. We use the volume growth assumption to carry out a scaling argument. The scaling appears to be unavoidable, therefore it seems that the only way to eliminate the volume growth restriction is to determine if stability or finite index, by themselves, imply a restriction on the volume growth.

In the proof of our theorem we will need the proposition and lemmas of the next section.

\section{AN UPPER BOUND FOR THE INDEX, AND SOME CONSEQUENCES OF FINITENESS OF THE INDEX}

Proposition. Let $n \geq 3$, and let $M^{n}$ be a minimally immersed oriented complete hypersurface in $R^{n+1}$. Then the index of $M^{n}$ satisfies the following upper bound,

$$
\operatorname{index}\left(M^{n}\right) \leq \omega_{n}^{-1}\left(\frac{\sqrt{e}(n-1) 2^{2 n+3}}{n-2}\right)^{n} \int_{M^{n}}|A|^{n} .
$$

Proof. This proposition follows immediately from Theorem 2 of $\mathrm{P} . \mathrm{Li}$ and S.-T. Yau in [3]. We only need to replace the Sobolev inequality for Euclidean space, that they use, by the Sobolev inequality for minimal submanifolds, see [4]. In fact, in their paper P. Li and S-T. Yau point out that their argument is also valid on manifolds on which a Sobolev inequality holds.

Remark. The method of P. Li and S.-T. Yau used above does not carry through for $n=2$. However, as mentioned in the introduction, a similar index estimate does hold for $n=2$ (see J. Tysk [8]).

For the sake of completeness we include the following well-known lemma. We give the proof as it is presented in [2].

Lemma 2. If $M$ has finite index there is a compact set $C$ so that $M-C$ is stable.

Proof. Recall that $D_{0}(\rho)$ denotes the geodesic ball centered at some fixed point 0 in $M$. Set

$$
\rho_{1}=2 \sup \left\{\rho: D_{0}(\rho) \text { is stable }\right\} .
$$

If $\rho_{1}$ is infinite, then $M$ is stable and we are done. Otherwise, let

$$
\rho_{2}=2 \sup \left\{\rho: D_{0}(\rho)-D_{0}\left(\rho_{1}\right) \text { is stable }\right\} .
$$

If $\rho_{2}$ is infinite the proof is complete. If not, we note that by the domain monotonicity for eigenvalues, $D_{0}\left(\rho_{2}\right)-D_{0}\left(\rho_{1}\right)$ is unstable. We then define, continuing this process, a strictly increasing sequence $\left\{\rho_{i}\right\}$ so that $D_{0}\left(\rho_{i}\right)-$ $D_{0}\left(\rho_{i-1}\right)$ is unstable for each $\rho_{i}$ in the sequence. The first eigenvalue for the stability operator $\Delta+|A|^{2}$, on $D_{0}\left(\rho_{i}\right)-D_{0}\left(\rho_{i-1}\right)$ is therefore negative. Let $f_{i}$ be the corresponding eigenfunction. Since the $f_{i}$ 's have disjoint support, they are linearly independent and the quadratic form associated to the second variation operator is negative definite on their linear span. Now, the index of $M$ is equal to the maximum dimension of a space on which the associated quadratic form 
is negative definite, so the assumed finiteness of the index of $M$ implies that there are only finitely many $f_{i}$ 's. The above construction must therefore stop after a finite number of steps and hence, $M$ is stable outside some compact set $C=D_{0}\left(\rho_{k}\right)$.

We will need the following lemma on the uniqueness of tangent planes at infinity for manifolds of finite index.

Lemma 3. Let $V^{n} \subset R^{n+1}$ be a connected minimal oriented n-dimensional submanifold of finite index, which is complete and properly immersed into the complement of some bounded open set in $R^{n+1}$. Assume that for any sequence $\left\{r_{i}\right\}$ of real numbers tending to infinity, the rescalings

$$
\frac{1}{r_{i}} V=\left\{\frac{1}{r_{i}} x: x \in V^{n} \subset R^{n+1}\right\},
$$

have a subsequence converging smoothly on $B_{0}(1)-\{0\}$, to a hyperplane of multiplicity one. Then this limiting hyperplane is unique, i.e. it does not depend on the sequence $\left\{r_{i}\right\}$.

Proof. To prove the uniqueness of the limiting hyperplanes we argue by contradiction: we assume that for some sequence of real numbers tending to infinity, $\left\{s_{i}\right\}_{i=1}^{\infty}$, the corresponding rescalings $\left\{\frac{1}{s_{i}} V\right\}_{i=1}^{\infty}$ converge to a hyperplane $\pi_{2}$, different from the limit $\pi_{1}$ of another sequence $\left\{\frac{1}{r_{i}} V\right\}_{i=1}^{\infty}$. Now let $K$ denote the closure of

$$
B_{0}\left(\frac{3}{4}\right)-B_{0}\left(\frac{1}{4}\right)
$$

and let $n_{1}$ be the normal of $\pi_{1}$ obtained as the limit of the normals to

$$
K \cap\left(\frac{1}{r_{i}} V\right) \text {. }
$$

Similarly, let $n_{2}$ be the normal of $\pi_{2}$ obtained as the limit of the normals to

$$
K \cap\left(\frac{1}{s_{i}} V\right)
$$

where we used the fact that the limiting hyperplanes have multiplicity one and the smooth convergence of the rescalings to guarantee that these normals converge. Now set $a=n_{1}-n_{2}$, and consider the function $V^{n} \ni p \rightarrow f(p)=$ $\left\langle e_{n+1}(p), a\right\rangle$, where $e_{n+1}(p)$ is the oriented unit normal of $V^{n}$ at $p$. For $i$ large enough, $f$ is positive on $\left(r_{i} K\right) \cap V$, and negative on $\left(s_{i} K\right) \cap V$. There therefore exists some bounded domain $D$ in $V-C$, so that $f$ is positive on $D$ and zero on the boundary of $D$, where $C$ is chosen in accordance with Lemma 2 so that $V-C$ is stable. We now recall the following well-known identity for minimal hypersurfaces, $\Delta f+|A|^{2} f=0$. The function $f$ is therefore an eigenfunction with eigenvalue zero of $\Delta+|A|^{2}$ on $D$ :

$$
\Delta f+|A|^{2} f=0 \text { on } D,\left.\quad f\right|_{\partial D}=0 .
$$


Hence, on some domain $D^{\prime}$ strictly containing $D$, the eigenvalue problem

$$
\Delta \phi+|A|^{2} \phi+\lambda \phi=0 \text { on } D^{\prime},\left.\quad \phi\right|_{\partial D^{\prime}}=0,
$$

has a negative eigenvalue by the domain monotonicity for eigenvalues. This means that $V-C$ is unstable, contradicting Lemma 2 , hence completing the proof of Lemma 3.

In our particular problem Lemma 3 has the following consequence.

Lemma 4. Let $M^{n}, n=3,4,5$, or 6 , be as in the statement of the theorem and with finite index. Then each end of $M^{n}$ has a unique tangent plane of multiplicity one at infinity.

Proof. Let $\left\{s_{i}\right\}_{i=1}^{\infty}$ be a sequence of real numbers tending to infinity and fix $x_{0} \in$ $R^{n+1}$. Since by the monotonicity formula for minimal varieties in Euclidean space, the function

$$
\frac{\operatorname{vol}(M \cap B(r))}{r^{n}}
$$

is nondecreasing in $r$, a subsequence of the rescalings

$$
M_{i}=\left\{s_{i}\left(x-x_{0}\right): x \in M\right\}, \quad i \geq 1,
$$

will, by standard Geometric Measure Theory, converge to a rectifiable current in any fixed ball centered at $x_{0}$. If the immersion of $M$ is not proper, and $x_{0}$ is chosen so that $M \cap B_{x_{0}}\left(r_{0}\right)$ is a noncompact subset of $M$ for all $r_{0}>0$, one can easily derive a contradiction to the assumed upper bound for the volume growth of $M$ from such convergent sequences of rescalings. Alternatively, use the isoperimetric inequality and the co-area formula to bound the volume of geodesic balls in $M$ from below, and then apply this bound to show that the immersion is proper. From Lemma 2, we know that $M-C$ is stable for some compact set $C$. From this stability, the properness of the immersion of $M$, and the assumed volume growth restriction, we can conclude using Theorem 3 by $\mathrm{R}$. Schoen and L. Simon [6], that $|A| \leq K_{1} / r$, where $K_{1}$ is some constant and $r$ the Euclidean distance function. Let $\left\{r_{i}\right\}_{i=1}^{\infty}$ be any seqeuence of real numbers tending to infinity. The homothetic rescalings

$$
\frac{1}{r_{i}} M=\left\{\frac{1}{r_{i}} x: x \in M^{n} \subset R^{n+1}\right\},
$$

are therefore minimal submanifolds whose second fundamental forms are uniformly bounded on any compact subset of $B_{0}(1)-\{0\}$. By the Smooth Compactness Theorem, see for instance $M$. Anderson [1], a subsequence of the rescalings $\left\{1 / r_{i} M\right\}$ therefore converges to minimal variety $\widetilde{M}$, which is smooth in $B_{0}(1)-\{0\}$. The proof of the Smooth Compactness Theroem is based on the fact that if the second fundamental form is uniformly bounded, then in balls whose radii depend only on $n$ and the bound on the second fundamental form, the components of minimal variety are graphs over their tangent planes. The estimate on the second fundamental form gives a $C^{1, \alpha}, \alpha<1$, bound on 
the solutions to the minimal surface equation over these balls. Elliptic regularity theory combined with the covering argument then gives the desired smooth convergence.

Combining this smooth convergence with the monotonicity formula for minimal submanifolds of Euclidean space, we obtain the well-known result that such a limit has the structure of cones on minimal codimension one submanifolds of $S^{n}$. Since $M-C$ is stable, these limiting cones also have to be stable. By the fundamental results of J. Simons [7], the only stable cones in $R^{4}, R^{5}, R^{6}$, and $R^{7}$, are in fact, hyperplanes. The tangent cones of $M^{n}$ at infinity are therefore tangent planes, possibly with multiplicities. In fact, using an argument appearing in [1], we can show that these multiplicities have to be one. Let $\pi$ be a limiting hyperplane with multiplicity $m>1$. Then for some $i$ large enough, one of the components of $1 / r_{i} M \cap \partial B_{0}(1)$ would, by the smooth convergence, provide a nontrivial covering of the equatorial sphere $\pi \cap \partial B_{0}(1)$, which is not possible since the dimension of this sphere is at least two.

By the monotonicity formula for minimal varieties, we see that the assumed volume growth restriction shows that there are only finitely many limiting tangent planes. By abuse of notation, let $\left\{1 / r_{i} M\right\}$ converge smoothly on $B_{0}(1)-$ $\{0\}$. As $i$ tends to infinity any connected component of $1 / r_{i} M$ in $B_{0}\left(\frac{3}{4}\right)-$ $B_{0}\left(\frac{1}{4}\right)$, has to converge to precisely one of the limiting hyperplanes, by the smooth convergence. This argument can be repeated for any sequence of homothetic shrinkings of $M$. Outside some compact set, $M$ therefore consists of connected components, referred to as ends and denoted $\left\{V_{k}\right\}$, for which a subsequence of $\left\{1 / r_{i} V_{k}\right\}$ converges smoothly to one of the limiting hyperplanes. Note that the number of ends is finite, since the argument above can be applied to every end, showing that each end has a subsequence of rescalings converging to some limiting hyperplane of which, as we have seen, there are only finitely many, each with multiplicity one. The uniqueness of the tangent plane of any end at infinity now follows from Lemma 3, completing the proof.

\section{Proof of THE Theorem}

Combining the proposition and lemmas of the preceding section we obtain the proof of the theorem in the following way.

Proof of the Theorem. If $\int_{M}|A|^{n}<\infty$, the proposition shows that the index of $M^{n}$ is finite.

Conversely, if the index of $M^{n}$ is finite we know from Lemma 4, that $M^{n}$ has unique tangent planes of multiplicity one at infinity. Also we remarked that outside some compact set in $R^{n+1}, M^{n}$ consists of finitely many ends. Since, as we showed above, $M^{n}$ is properly immersed, we know that only a compact set in $M^{n}$ can be included in a compact set in $R^{n+1}$, so we need only prove that the integral of $|A|^{n}$ over the ends is finite. Now, since there are only finitely many ends, we need only show that the integral over any end is finite. 
Since the ends of $M^{n}$ converge to hyperplanes of multiplicity one, we can conclude that outside some compact set, each end is a graph of bounded slope over the tangent plane at infinity. According to Proposition 3 by R. Schoen [5], this means that on the ends the second fundamental form satisfies the estimate $|A| \leq K_{2} / r^{n}$, for some constant $K_{2}$. Since the ends converge to hyperplanes of dimension $n$, we clearly have $\int_{V}|A|^{n}<\infty$, for all ends $V$, showing that $\int_{M^{n}}|A|^{n}<\infty$, thus completing our proof.

\section{REFERENCES}

1. M. Anderson, The compatification of a minimal submanifold in Euclidean space by the Gauss map (to appear).

2. D. Fischer-Colbrie, On complete minimal surfaces with finite index in three manifolds, Invent. Math. 82 (1985), 121-132.

3. P. Li and S.-T. Yau, On the Schrödinger equation and the eigenvalue problem, Comm. Math. Phys. 88 (1983), 309-318.

4. J. H. Michael and L. Simon, Sobolev and mean value inequalities on generalized submanifolds of $R^{n}$, Comm. Pure Appl. Math. 26 (1973), 361-379.

5. R. Schoen, Uniqueness, symmetry, and embeddedness of minimal surfaces, J. Differential Geom. 18 (1983), 791-801.

6. R. Schoen and L. Simon, Regularity of stable minimal surfaces, Comm. Pure Appl. Math. 34 (1981), 741-797.

7. J. Simons, Minimal varieties in Riemannian manifolds, Ann. of Math. 88 (1968), 62-105.

8. J. Tysk, Eigenvalue estimates with applications to minimal surfaces, Pac. J. Math. 128 (1987), 361-366.

Department of Mathematics, Columbia University, New York, New York, 10027 